\title{
LA FAUNA CHAQUEÑA DE CÓRDOBA (ARGENTINA) AFECTADA POR LA TRANSFORMACIÓN AGRÍCOLA
}

\section{THE CHACOAN FAUNA OF CORDOBA (ARGENTINA) AFFECTED BY THE AGRICULTURAL TRANSFORMATION OF THE HABITAT}

\author{
Cintia Cebollada Pütz, Mariana Basso, Estefanía Ruiz de los Llanos y Maura Kufner ${ }^{1}$
}

\begin{abstract}
Resumen
En el Chaco cordobés la agricultura intensiva ha simplificado el hábitat y empobrecido la biodiversidad. El Departamento Totoral en el centro provincial, consiste en una matriz agrícola con escasos relictos de bosque. La incidencia de la transformación agrícola del hábitat sobre atributos de las comunidades de vertebrados, se estudió en remanentes de vegetación nativa con distinta condición y forma (hábitat cuadrangular HC y alongado HA). La estructura del hábitat, aún con influencia climática, fue más completa en el primero; en HA se comprobó ausencia de estratos superiores a $7 \mathrm{~m}$, arbustización y aumento de suelo desnudo. Se anotó un total de 109 especies; durante el período húmedo hubo mayor número de especies y abundancia de individuos. Las alteraciones del hábitat favorecieron especies de distribución amplia, de agrosistemas, y empobrecimiento faunístico general. La avifauna mostró reemplazo estacional de especies, no así la herpeto y mastofauna, comunidades más permanentes. La estructura gremial presentó mayoría de insectívoros. La diversidad de herpetofauna fue mayor en la época húmeda, en micromamíferos no mostró diferencia estacional y la de avifauna fue varios órdenes mayor en ambas estaciones. La distribución de la herpetofauna y mastofauna en el hábitat se relacionó principalmente con buena cobertura gramíneo-herbácea, y la de aves con los estratos altos del bosque. Las variables del hábitat perturbadas por presión humana inciden sobre los valores de importancia de las especies, las fluctuaciones estacionales, los movimientos espaciales y favorece a ciertos grupos. Los relictos de vegetación nativa revisten importancia para preservación y restauración de la fauna regional.
\end{abstract}

Palabras clave: Chaco Cordobés, expansión agrícola, fauna silvestre, hábitat degradado, conservación.

Intensive agriculture has simplified the Chaco habitat and impoverished its biodiversity, in Córdoba, Argentina. Thus, the district of Totoral in the provincial core has become a cultivated matrix with very few relicts of native forest. The impact of agricultural transformation of the habitat, on vertebrate communities attributes, was studied in remnants of native vegetation with different condition and shape (quadrangular HC, and elongated HA). Habitat structure was more complete in the first one, even considering climatic influence; while in HA strata higher than $7 \mathrm{~m}$ were missing, and bare soil and shrub thicket were increased. A total of 109 species was scored. Species and individuals were more abundant during the wet period. Alterations of habitat favored: species of wide distribution, species tolerant to agro-systems conditions, and a general faunal impoverishment. Birds showed seasonal species replacement, but not in more permanent herpetological and mammalian communities. The guild structure presented most insectivores. During wet season herpetological specific diversity was higher, but in mammals it showed no seasonal difference, whereas in birds it was several orders higher in both seasons. The distribution of the herpetic and mammal communities in the habitat was mainly associated with coverage of herbaceous layer, while distribution of bird communities was related mostly to higher forest strata. Human pressures disturbing habitat characteristics in the Cordoban Chaco, affect important parameters and seasonal fluctuations of species, their spatial movements, as well as favor certain vertebrate groups. Forest remnants are essential for preservation and restoration of the regional fauna.

Key words: Cordoba Chaco, agricultural expansion, degraded habitat, wildlife conservation.

\section{Introducción.}

El Chaco concentra en América del Sur gran diversidad de elementos florísticos y faunísticos, así como una heterogeneidad de ambientes y ecotonos. Ésta es dada en horizontal por el mosaico de isletas de bosque, sabanas, pastizales, lagunas, ríos y arroyos 
(Morello \& Matteucci, 1999) y en vertical por estratos herbáceo, arbustivo y arbóreo, lianas, epífitas, etc. (Luti et al., 1979). La relación entre esa complejidad de hábitat y la diversidad de las comunidades animales, ha sido señalada en bosques semiáridos de Argentina (Altrichter et al., 2004; Briguera et al., 2005, entre otros).

El área chaqueña ha recibido durante varias décadas los impactos de la ganadería, la agricultura y el aprovechamiento forestal. En particular, las amenazas mencionadas para el Bosque Chaqueño son el aumento de la superficie agrícola, la presión de productores y campesinos sobre el recurso forestal y los incendios rurales (Adámoli et al., 2004).

La provincia de Córdoba no ha sido ajena a este proceso; el paisaje chaqueño original ha sido modificado, dando lugar a tierras culturales principalmente de soja y otros granos-, a bosques secundarios y arbustales de substitución. El bosque ha quedado representado por remanentes aislados, relegados a sitios no aptos para la agricultura (Zak \& Cabido, 2002). A partir de los años 70 el área central de la provincia ha sido muy impactada por agricultura -en particular el Departamento Totoral- cuyo desmonte, autorizado por la administración provincial entre 1979 y 2004, ha dejado relictos boscosos escasos y en distinta condición (Bazán, 1998; Stamatti et al., 2004).

La pérdida del hábitat ocasiona cambios en las relaciones ecológicas que influyen en la organización del sistema, en las cadenas tróficas y en las relaciones interespecíficas (Fahrig, 2003). Las modificaciones en la estructura y disponibilidad del hábitat y la distancia entre los remanentes, alteran los intercambios y flujos de especies (Bennet, 1990; Angelstam, 1992), a la vez que inciden en atributos de las comunidades faunísticas, tales como la composición, abundancia y diversidad (Ubeda \& Griguera, 2003).

En el área de Totoral, dadas las condiciones del hábitat mencionadas, se esperaría desaparición de elementos de la biodiversidad chaqueña y cambios en atributos de la fauna. Por ejemplo, que hubiera ciertas especies muy abundantes favorecidas por los cultivos, mayor número de granívoras e insectívoras y de vertebrados de áreas abiertas, en razón de abundante estrato gramíneo-herbáceo de tipo pampeano, prevalencia de especies generalistas. Estas hipótesis se abordan a partir del análisis de la estructura, distribución y condición de la vegetación nativa en relictos de bosque, de atributos de las comunidades terrestres de la fauna, y de su relación con el hábitat degradado por actividad agrícola, en el centro de la provincia de Córdoba, Argentina.

\section{Materiales y métodos.}

Área de estudio.

El Bosque Chaqueño Oriental en la provincia de Córdoba se distribuye al E de las sierras del Norte y presenta características florísticas y fisonómicas relacionadas con precipitaciones entre 550 y $600 \mathrm{~mm}$ anuales. El bosque, con sus estratos arbóreo, arbustivo y herbáceo, constituye la formación climácica de mayor desarrollo. La variedad fisonómica, está dada por subclimax edáficos (cardonales, salinas, bañados, etc.). El estrato arbóreo superior (8 a $>10 \mathrm{~m}$ ) esta constituido principalmente por el "quebracho blanco" (Aspidosperma quebracho-blanco) y "algarrobo negro" (Prosopis nigra); el "quebracho colorado" (Schinopsis lorentzii) de mayor porte, se reduce a distribuciones locales. El estrato arbustivo presenta entre otras "garabato" (Acacia praecox), "moradillos" (Schinus spp.) y "jarilla” (Larrea divaricata), mientras que en el estrato herbáceo predominan gramíneas como Trichloris spp., Setaria spp. y Nassella spp. (Luti et al., 1979).

El estudio se realizó en la estancia La Esperanza localizada en la zona rural del Departamento Totoral, aproximadamente $5 \mathrm{~km}$ al $\mathrm{S}$ de la localidad de Las Peñas y $100 \mathrm{~km}$ hacia el NE de la capital provincial. Allí, los procesos de intervención humana han originado una matriz agrícola principalmente de soja, y rotaciones con cultivos de trigo en otoño y maíz en primavera. En dicha matriz se distribuyen relictos de bosque con forma y tamaño variado y en distintas etapas sucesionales.

Metodología.

La distribución y estructura del hábitat se analizó en escala de paisaje a fin de proporcionar el contexto regional para los sitios de estudio. Los componentes paisajísticos se describieron mediante conteo de píxeles (Matteucci, 1998) sobre una imagen satelital de escala 1:245 (Google Earth, 2006). En el nivel local, se analizó la situación de la vegetación en dos relictos boscosos de forma cuadrangular (HC) y alongada (HA), considerados representativos de otros remanentes chaqueños incluidos en la matriz agrícola. Se estimó la cobertura vegetal -horizontal y por intervalos de altura- según la Línea de Canfield (Hays et al., 1981) en los períodos estacionales seco y húmedo.

Las mediciones se hicieron estacionalmente sobre dos líneas de transecta de $30 \mathrm{~m}$ de longitud, ubicadas en cada hábitat, a partir de $50 \mathrm{~m}$ del borde. En cada línea se midió la cobertura vegetal vertical de los estratos comprendidos entre el suelo $(0.01 \mathrm{~m})$ y el dosel superior $(>7 \mathrm{~m})$. La cobertura vegetal por estaciones y en cada hábitat, se comparó mediante la prueba de $X^{2}$ de Pearson (Siegel, 1980). Para investigar la relación de la fauna con el hábitat, los datos de cobertura vegetal se clasificaron en cuatro intervalos de altura funcionalmente importantes: estrato herbáceo (0.01-0.5 m), arbustivo (0.51-2 m), ramaje y copa baja (2.01-4 m), copa alta $(4.01-7 \mathrm{~m})$ y dosel superior $(>7 \mathrm{~m})$

La composición faunística se estudió a lo largo de un ciclo biológico, entre septiembre de 2005 y octubre 
de 2006. El relevamiento de campo se realizó en base a líneas de trampeo y puntos de observación, siguiendo un patrón regular (Matteucci \& Colma, 1982). Los tetrápodos de tierra (anfibios, reptiles y pequeños mamíferos) se colectaron mediante 60 trampas de caída, ubicadas en 10 líneas cada una de 50 $\mathrm{m}$, disponiendo cinco en cada hábitat. La recolección se efectuó cada 45 días aproximadamente. Los especímenes capturados se depositaron en la Colección Biológica del CERNAR (CBCVertebrados, 2011). El muestreo de aves se desarrolló estacionalmente según la metodología de puntos de observación (Bibby et al., 1992). En seis puntos en total establecidos mediante GPS se anotaron todos los individuos registrados durante cinco repeticiones de 15 minutos cada una.

La identificación de las especies de anfibios y reptiles se hizo siguiendo a Lavilla et al. (1992) y Cabrera (2001), la de aves a Narosky \& Yzurieta (2003) y la de micromamíferos a Polop \& Busch (2010).

Los datos sobre composición y abundancia de los tetrápodos se analizaron por períodos estacionales y por hábitat, mientras que los de la avifauna se compararon sólo estacionalmente debido a su amplio rango de uso del hábitat que sobrepasa el límite de los fragmentos boscosos.

En base a la principal característica de su dieta, las especies se agruparon en los siguientes gremios tróficos: Omnívoro, Granívoro, Carnívoro, Carroñero, Frugívoro-granívoro, Nectarívoro, Insectívoro y Herbívoro (Gallardo, 1987; Giannoni et al., 2005; Giraudo et al., 2006 y otros).

La diversidad faunística se estimó mediante el índice de Shannon-Wiener ( $H^{\prime}$ '), evaluándose posibles diferencias mediante el test " $t$ " de Hutchinson. Complementariamente, se calculó la similitud de las comunidades faunísticas presentes en los dos hábitat, por medio del índice cuantitativo de Sorenson $\left(\mathrm{C}_{\mathrm{N}}\right)$ (Magurran, 1988; Gordon, 1997).

Uso del hábitat: la distribución de tetrápodos del suelo (anfibios, reptiles y mamíferos) en relación con la cobertura herbácea, dada su importancia para el grupo, se analizó a través de regresiones simples considerando un análisis general para el total de tetrápodos, donde la variable predictora (x) fue el porcentaje de cobertura del estrato herbáceo y la variable respuesta (y) el número de especies. Análisis particulares se realizaron para la herpetofauna y los micromamíferos; en este caso la variable respuesta (y) fue el promedio de individuos por especie (Manzanilla \& Péfaur, 2000; Altrichter et al., 2004).

Por su parte, la distribución de la avifauna en relación con la estructura del hábitat se investigó a partir de regresiones múltiples en las cuales las variables predictoras $\left(\mathrm{x}_{1}, \mathrm{x}_{2}\right)$ fueron la altura $\mathrm{y}$ el porcentaje de cobertura de los estratos de vegetación, y la variable respuesta (y) fue el número de especies (Carrascal \& Palomino, 2005).

\section{Resultados.}

El hábitat de bosque. Distribución y estructura.

A nivel de paisaje se identificaron tres unidades, con predominio del área cultivada (87.59\%) lo cual confirió a ésta la condición de "matriz". Las otras unidades reconocidas tuvieron menor representación en el paisaje: la superficie de vegetación nativa $6.73 \%$ (correspondiente al Bosque Chaqueño) fue similar a la del área urbanizada (5.86\%).

La fisonomía de la vegetación reveló al quebracho blanco como la especie de mayor porte $(6-7 \mathrm{~m}) \mathrm{y}$ al algarrobo negro como la dominante, y algunas especies indicadoras de sobrepastoreo (Malvastrum coromandelianum, Sphaeralcea cordobensis y Cestrum parqui).

En los dos hábitats (HC y HA) la vegetación mostró diferente condición y fisonomía (Fig. 1). En el primero la cobertura herbácea fue baja, la arbustiva variable a lo largo del año, y el estrato arbóreo alcanzó los $7 \mathrm{~m}$. En el segundo hábitat los estratos herbáceo y arbustivo estuvieron comparativamente más desarrollados, mientras que el arbóreo se presentó raleado y con menor altura (aproximadamente $4 \mathrm{~m}$ ). La estructura de la vegetación de estos dos hábitats resultó diferente, tanto en el período seco como en el húmedo $(\mathrm{p}<0.001)$.

La estacionalidad chaqueña se manifestó en cambios significativos de la estructura de la vegetación en los dos hábitats (Fig. 1). El hábitat HC mostró en el período húmedo una distribución más equitativa de la cobertura entre los estratos verticales de la vegetación, mientras que en el período seco disminuyeron la cobertura arbórea y herbácea y aumentó la arbustiva $(\mathrm{p}<0.001)$. Por su parte, el hábitat HA presentó en el período seco aumento de la cobertura herbácea y arbustiva (hasta $1 \mathrm{~m}$ ) y disminución de la arbórea $(\mathrm{p}<0.001)$.

Atributos de las comunidades faunísticas.

Los vertebrados estuvieron representados en el área de estudio por 3 familias de anfibios, 6 de reptiles, 34 de aves y 9 de mamíferos, registrándose en total 109 especies (Tablas 1, 2, 3). La influencia de la estacionalidad chaqueña se evidenció en un mayor número de especies (S) y abundancia de individuos (N) durante el período húmedo $(\mathrm{S}=93 ; \mathrm{N}=1003)$, en relación con el período seco $(S=67 ; \mathrm{N}=848)$.

En referencia a la abundancia de cada grupo, en la herpetofauna se destacó Rhinella arenarum (44\% en el período húmedo y $84 \%$ en el período seco), seguido por Physalaemus biligonigerus $(18 \%$ y 5\%, respectivamente) (Tabla 1). Por su parte, en la avifauna Columbina picui, Coragyps atratus y Myiopsitta monachus sumadas, aportaron $41 \%$ del total de individuos, en ambas estaciones. Un caso 
Tabla 1. Comunidades de anfibios y reptiles en los hábitats de bosque. Composición y abundancia estacional y gremios tróficos.

\begin{tabular}{lccccc}
\hline Nombre específico & $\begin{array}{c}\text { Gremio } \\
\text { trófico }\end{array}$ & \multicolumn{2}{c}{$\begin{array}{c}\text { Período } \\
\text { Húmedo }\end{array}$} & \multicolumn{2}{c}{ Período } \\
& & HC & HA & HC & HA \\
\hline Chaunus arenarum & I & 21 & 31 & 12 & 36 \\
C. schneideri & I & - & 2 & - & 2 \\
Leptodactylus gracilis & I & 3 & 5 & - & 1 \\
L. mistacinus & I & 3 & 9 & - & - \\
Physalaemus biligonigerus & I & 7 & 14 & 1 & 2 \\
Chelonoidis petersi & H & 1 & - & - & - \\
Mabuya dorsivitatta & I & - & 6 & - & 1 \\
Cnemidophorus sp. & I & 1 & 1 & - & - \\
Teius oculatus & I & 2 & 3 & - & 1 \\
T. teyou & I & 2 & - & - & - \\
Cercosaura schreibersii & I & 1 & 12 & 1 & 1 \\
Leptotyphlops albipuntus & I & - & 1 & - & - \\
Lystrophis semicinctus & C & - & 1 & - & - \\
Total de individuos & & 41 & 85 & 14 & 44 \\
Número de Especies & & 9 & 11 & 3 & 7 \\
\hline
\end{tabular}

Referencias. Gremio trófico: H, Herbívoro; I, Insectívoro; C, Carnívoro. HC: Fragmentos de Bosque Cuadrangulares, HA: Fragmentos de Bosque Alongados.

particular fue el de Zenaida auriculata que se desplazaba en grandes bandadas (>300 individuos) dificultándose la estimación de su abundancia; debido a ello se la descartó de los análisis cuantitativos (Tabla 2). Entre los micromamíferos, el complejo Calomys laucha-musculinus fue el más abundante (54\% en el período húmedo y $47 \%$ en el período seco), seguido por C. venustus (22\% y 30\%). Los mamíferos de mayor tamaño presentaron otras seis especies, aunque en números bajos (Tabla 3).

La clasificación de la fauna en gremios tróficos, indicó que los insectívoros estuvieron mejor representados (47\%), seguidos por omnívoros y granívoros (17\% y $12 \%$, respectivamente). En cada uno de los gremios restantes (Granívoro-insectívoro, Carnívoro, Carroñero, Frugívoro-granívoro, Nectarívoroinsectívoro y Herbívoro) la representación fue menor del $10 \%$. La comunidad de herpetofauna estuvo constituida sólo por especies insectívoras; mientras que la de mamíferos se integró de herbívoros y omnívoros. La comunidad de aves, con especies de todos los gremios, presentó la mayor amplitud trófica.

La similitud de la composición faunística general, hallada entre períodos estacionales húmedo y seco $\left(\mathrm{C}_{\mathrm{N}}=0.65\right)$, se debió especialmente a las comunidades de herpetofauna y de mamíferos, ya que la de aves fue la comunidad con mayor reemplazo estacional de especies.

En cuanto a la diversidad específica de la fauna, la misma no mostró en general influencia de la estacionalidad entre período húmedo y seco $\left(\mathrm{H}_{\mathrm{PH}}^{\prime}=3.34 ; \mathrm{H}_{\mathrm{PS}}^{\prime}=3.31 ; \mathrm{P}=0.09\right)$, con la excepción de la comunidad de anfibios y reptiles (Tabla 4). En efecto, la diversidad de la herpetofauna resultó significativamente mayor $(p<0,001)$ en la época húmeda. En cuanto a la diversidad de micromamíferos, si bien no acusó diferencia estacional $(\mathrm{p}=0.26)$, en el período seco se constató ausencia de dos especies: Graomys griseoflavus y Oxymicterus rufus. La comunidad de aves fue en general más diversa que las de tetrápodos. Entre estaciones la diversidad aviar no presentó variaciones, aunque en la época estival se registró mayor número de aves (Tabla 4) y el reemplazo estacional de especies antes citado. En este sentido, 23 especies se presentaron sólo en el período húmedo y 15 sólo en el seco.

Distribución de la fauna en relación con la estructura del hábitat.

La distribución en el hábitat del número de individuos de tetrápodos fue mayor en el hábitat alongado (HA) que en el cuadrangular (HC), lo cual respectivamente se manifestó en 129 y 55 individuos de la herpetofauna, y en 294 y 107 individuos de la mastofauna. Las aves con amplias áreas de acción- hicieron un uso generalizado del hábitat.

La relación entre la riqueza de tetrápodos y la estructura del hábitat arrojó una correlación positiva entre el número de especies y la cobertura del estrato herbáceo (hasta $0.50 \mathrm{~m}$ ), según el modelo cuadrático $\mathrm{y}=-0.03 \mathrm{x}^{2}+1.40 \mathrm{x}-0.84\left(\mathrm{R}^{2}=0.80 ; \mathrm{p}<0.05\right) . \quad$ El

Tabla 3. Comunidades de mamíferos en los hábitats de bosque. Composición y abundancia estacional y gremios tróficos.

\begin{tabular}{lccccc} 
Nombre específico & $\begin{array}{c}\text { Gremio } \\
\text { Trófico }\end{array}$ & \multicolumn{2}{c}{$\begin{array}{c}\text { Período } \\
\text { Húmedo }\end{array}$} & \multicolumn{2}{c}{$\begin{array}{c}\text { Período } \\
\text { Seco }\end{array}$} \\
\cline { 3 - 6 } & & HC & HA & HC & HA \\
\hline Thylamys pallidior & I & 5 & - & 4 & - \\
Didelphis albiventris & $\mathrm{O}$ & - & 1 & - & - \\
Chaetophractus sp. & $\mathrm{O}$ & 1 & - & - & - \\
$\begin{array}{l}\text { Dasypus hybridus } \\
\text { Akodon sp. }\end{array}$ & $\mathrm{O}$ & - & - & - & 1 \\
$\begin{array}{l}\text { Oxymycterus rufus } \\
\text { Oligoryzomys flavescens }\end{array}$ & $\mathrm{H}$ & 9 & 24 & 5 & 13 \\
Calomys laucha- & $\mathrm{O}$ & - & 3 & - & - \\
musculinus & $\mathrm{G}$ & 45 & 80 & 12 & 53 \\
C. venustus & & & & & \\
$\begin{array}{l}\text { Graomys centralis } \\
\text { Cricetidae s/d }\end{array}$ & $\mathrm{O}$ & 8 & 43 & 5 & 37 \\
$\begin{array}{l}\text { Ctenomys sp. } \\
\text { Conepatus sp. }\end{array}$ & $\mathrm{H}$ & - & 1 & - & - \\
$\begin{array}{l}\text { Pseudalopex } \\
\text { gymnocercus }\end{array}$ & $\mathrm{H}$ & - & 1 & - & - \\
$\begin{array}{l}\text { Mazama gouazoubira. } \\
\text { Total de individuos }\end{array}$ & $\mathrm{I}$ & 1 & - & - & - \\
Número de Especies & $\mathrm{O}$ & - & 1 & - & 1 \\
\hline
\end{tabular}

Referencias: H Herbívoro; G Granívoro; O Omnívoro; I Insectívoro; Ca Carroñero. HC: Hábitat de Bosque Cuadrangular, HA: Hábitat de Bosque Alongado. S/d: sin determinar. 
cálculo del vértice de la curva $\left(\mathrm{x}_{\mathrm{v}} ; \mathrm{y}_{\mathrm{v}}\right)$ estimó el mayor número de especies $(\mathrm{N}=15.5)$ con coberturas herbáceas de 23\% en este hábitat (Fig. 2).

\section{Discusión.}

El hábitat de bosque. Distribución y estructura

En un paisaje donde domina la matriz de cultivos, el bosque nativo ha quedado confinado en fragmentos aislados. Esta situación induce cambios en las relaciones ecológicas del sistema, modifica la organización, interrumpe los intercambios y flujos de especies, etc. (Fahrig, 2003).

Los fragmentos boscosos estudiados se diferencian en gran medida de la estructura descripta para el Bosque Chaqueño Oriental de Córdoba (Luti et

Los análisis particulares mostraron relación positiva de la riqueza faunística con el estrato herbáceo. Con respecto a la herpetofauna, un modelo de regresión cuadrático explicó el $73 \%$ de los datos observados según la siguiente ecuación $y=-0.02 \mathrm{x}^{2}+$ $0.73 x-0.67(p<0.05)$. Es decir, la cobertura gramíneo-herbácea cercana al 18\% favorecería mayor abundancia $(n=6)$ de anfibios y reptiles. Por su parte, en los micromamíferos la relación positiva con dicho estrato -en ambos hábitats- no fue significativa ( $\mathrm{y}=$ $\left.0.03 \mathrm{x}^{2}+1.41 \mathrm{x}-3.27 ; \mathrm{p}=0.27\right)$; los números mayores de roedores corresponderían a coberturas herbáceas mayores a $25 \%$.

Se comprobó la relación entre la distribución espacial vertical de las aves y la estructura del hábitat y su condición. Por una parte, el número de especies se relacionó de forma positiva y significativa $(\mathrm{P}=$ 0.047) con la altura $\left(\mathrm{x}_{1}\right)$ y con el porcentaje de cobertura $\left(\mathrm{x}_{2}\right)$ de los estratos de vegetación; el modelo de regresión obtenido $\left(\mathrm{y}=3.3 \mathrm{x}_{1}-8.6\left(\mathrm{x}_{2}\right)^{2}+22.65\right)$ explicó el $72 \%$ de los datos observados. Considerando los períodos estacionales, en el período húmedo este análisis explicó el 89\% de la variabilidad en el número de especies de aves, según la ecuación siguiente: $\mathrm{N}$ de especies $=2.7 \mathrm{x}_{1}$ (m de altura de vegetación $)-0.07 \mathrm{x}_{2}$ $(\%$ cobertura vegetal $)+9.85$. En el período seco -en que disminuye la fronda en las alturas superiores a 4 m- cobró importancia el estrato de Ramaje y Copa baja (ajuste 26\%) (Tabla 5). Adicionalmente, el análisis por pasos (stepwise) permitió identificar la Altura de la vegetación como la variable del hábitat más importante.

Tabla 5. Estructura del hábitat y distribución de la avifauna. Variables predictoras ( $\mathrm{X}_{1}$ Altura de la vegetación; $\mathrm{X}_{2} \%$ Cobertura vegetal) y respuesta (Y Número de especies de aves) para los análisis de regresión múltiple.

\begin{tabular}{lcccc}
\hline \multirow{2}{*}{$\mathrm{X}_{1}$ Altura de la vegetación $(\mathrm{m})$} & \multicolumn{2}{c}{ Período Húmedo } & \multicolumn{2}{c}{ Período Seco } \\
\cline { 2 - 5 } & $\mathrm{X}_{2}$ & $\mathrm{Y}$ & $\mathrm{X}_{2}$ & $\mathrm{Y}$ \\
\hline Copa alta 4,01-7 & 22,21 & 25 & 9,81 & 14 \\
Ramaje y copa baja 2,01-4 & 19,33 & 23 & 24,86 & 27 \\
Arbustivo 0,51-2 & 58,46 & 12 & 65,33 & 5 \\
Herbáceo 0,01-0,5 & 18,29 & 9 & 3,35 & 6 \\
\hline
\end{tabular}

al., 1979; Zak \& Cabido, 2002) debido a factores antrópicos como: desmonte, avance de la frontera agrícola, sobrepastoreo, ramoneo de renovales, compactación del suelo, etc. (Adámoli et al., 2004). Las alteraciones en la estructura (ausencia de estratos arbóreos superiores, arbustización, denudación observada del suelo) fueron más notorias en el hábitat de forma alongada (HA), presentando mejor condición el hábitat cuadrangular (HC). Fragmentos con mayor relación de borde están más expuestos a percolación hacia el interior de la degradación circundante (Saunders et al., 1991; Angelstam, 1992). En ambos tipos de hábitat, sin embargo, se evidenciaron cambios propios de la estacionalidad chaqueña típica en la cobertura de la vegetación remanente.

Atributos de las comunidades faunísticas

Composición. La fauna se compone en su mayoría de especies de áreas abiertas, en relación con la condición del hábitat. Entre los anfibios y reptiles se cuentan el sapo buey ( $R$. schneideri), asociado a gramíneas o pajonales de hasta un metro de altura (Cei, 1986; di Tada et al., 1996) y el lagarto mabuya (M. dorsivitatta) de pastizales cortos con vegetación leñosa escasa, semejante a la de su distribución en el Espinal y la Pampa (Cei, 1986; Cabrera, 1996). La composición de mamíferos -principalmente roedores cricétidos- fue similar a la de agrosistemas pampeanos (Polop \& Sabatini, 1993; Bilenca \& Kravetz, 1995), probablemente en relación con la "pampización" antrópica del hábitat (Kufner et al., 2005). En cuanto a la avifauna, su composición es chaqueña en una proporción mayor al 50\% (Narosky \& Yzurieta, 2003).

Abundancia. Las especies de amplia distribución y requerimientos de hábitat poco específicos aportaron los mayores números y/o persistencia. Por ejemplo, en la herpetofauna, el cosmopolita sapo común $(R$. arenarum) y la ranita llorona $(P$. biligonigerus) distribuida en toda la provincia, son abundantes y con capacidad de adaptación y penetración en ambientes modificados (Cei, 1980; Peltzer, et al. 2005). La característica de una comunidad de 
anfibios anuros con pocas especies y gran número de individuos, comprobada en nuestro estudio, es citada para otros ecosistemas agrícolas (Peltzer et al., 2004). En las aves, la abundancia de algunas especies como la paloma torcaza (Z. auriculata) y los tordos (Molothrus spp.) -además de su distribución generalizada (Narosky \& Yzurieta, 2003)- se relaciona con la capacidad de adaptación a sistemas agrícolas y el aprovechamiento oportunista de cualquier aumento en la disponibilidad de comida, lo cual favorece rápidos incrementos poblacionales (Giraudo et al., 2006). Entre los mamíferos, la prevalencia del complejo Calomys, según se dijo, es semejante a la mencionada para $C$. musculinus en bordes de cultivos de agrosistemas pampeanos (Polop \& Sabatini, 1993; de Villafañe et al., 1994). Los hábitats que bordean los cultivos en los que predomina el estrato herbáceo (como HA) constituyen sitios favorables (Polop et al., 1985), a juzgar por la mayor abundancia de roedores registrada allí. De persistir las condiciones descriptas sería dable esperar un aumento en la probabilidad de trasmisión del virus Junín, causante de la Fiebre Hemorrágica Argentina (González Ittig \& Gardenal, 2004).

En síntesis, en cuanto a composición y abundancia, las alteraciones a nivel de paisaje y del hábitat local, favorecieron a las especies de distribución amplia y requerimientos poco específicos de hábitat y a especies de agrosistemas, así como a un empobrecimiento general de la fauna, en relación con otras áreas chaqueñas (Briguera et al., 2005; Kufner et al., 2005; Giraudo et al., 2006).

Gremios tróficos. La organización gremial de la fauna fue la esperada para un sistema chaqueño, en cuanto predominaron las especies insectívoras, acorde a una provisión constante de artrópodos (Costa Gorriz \& Bucher, 1995). Algunas especies granívoras son beneficiadas por el tipo de uso de la tierra que genera un paisaje "en mosaico", alternando parches de bosque y grandes parcelas de cultivo. Las condiciones son muy propicias para especies gregarias, con gran movilidad y alta tasa reproductiva, como Z. auriculata y M. monachus (Bucher, 1980; Bucher, 1990). En suma, en la estructura gremial de la fauna del área de estudio -si bien aún se mantienen características semejantes a la del Bosque Chaqueño- es insoslayable la fuerte incidencia de una matriz agrícola de granos que potencia numéricamente a ciertas entidades.

Diversidad específica. Teniendo en cuenta el total de vertebrados del área, la diversidad no evidenció cambios estacionales, ni en relación con el hábitat, salvo casos particulares que se exponen a continuación. En cuanto a la diversidad de tetrápodos (herpeto y mastofauna), la estructura del hábitat no influyó en esta variable; los dos hábitats (HA y HC) presentarían semejante condición para la fauna, y la influencia de la matriz es importante. Con respecto a la avifauna, comunidad con el mayor peso específico y numérico, sus ensambles utilizan ampliamente hábitats con fisonomías diferentes.

En relación con los cambios estacionales, la herpetofauna fue la única comunidad que acusó incidencia estacional en la diversidad. En el período húmedo la cobertura, la estructura de la vegetación y las características hídricas del ambiente favorecen a las comunidades de anfibios y reptiles. Las variaciones estacionales encontradas en las mismas revelan tanto la persistencia del ciclo natural chaqueño, como su sensibilidad ante alteraciones del ambiente reproductivo (Briguera et al., 2006).

La diversidad de la mastofauna acusó menor influencia estacional, aunque el número de individuos de las especies disminuyó en el período seco invernal. Esta situación es diferente a lo esperado en los ciclos poblacionales (aumentos en otoño-invierno) de micromamíferos chaco-pampeanos (Zuleta et al., 1988; Kufner et al., 2005; Polop \& Busch, 2010) y podría relacionarse con la condición degradada del medio. No se puede descontar el probable impacto de fumigaciones con agroquímicos sobre cultivos adyacentes, en épocas de reproducción de estas especies.

La elevada diversidad aviar no difirió entre estaciones y la similitud observada evidencia un reemplazo de especies. Un aumento en la diversidad aviar se hubiera esperado considerando los cambios estivales en la estructura del hábitat y la disponibilidad de alimento en bosques templados (Dardanelli \& Nores, 2001). La avifauna de estos bosques suele tener buena capacidad de colonización por lo que el arribo de especies migrantes y los movimientos locales que realizan las aves residentes (Codesido \& Bilenca, 2004), contribuirían a producir los reemplazos estacionales en la composición.

Distribución de la fauna en relación con la estructura del hábitat

La estructura horizontal y vertical de la vegetación influye en el uso del hábitat y en la distribución espacial de la fauna. Estudios de diversos autores sugieren que el tipo de vegetación, la modificación del paisaje, así como el grado de perturbación de los hábitats afectan a la fauna (Bucher et al., 2001; Dardanelli \& Nores, 2001). Algunas aves como Picoides mixtus, Melanerpes cactorum y Lepidocolaptes angustrirostris, que requieren áreas superiores a 45 y 100 ha, se ven afectadas por la disminución y fragmentación de su hábitat (Costa Gorriz \& Bucher, 1995). Y, a juzgar por los bajos números de ciertas poblaciones de mamíferos del bosque (ej.: T. pallidior y M. gouazoubira), éstas también están comprometidas (Miatello, 2007).

Factores destacados intervinientes en la relación entre la estructura del hábitat y la distribución de los tetrápodos, fueron la estacionalidad climática y la modificación antrópica. La influencia de la estacionalidad se constató en el aumento del número 
de especies durante el periodo húmedo, como es esperable en especial en la herpetofauna chaqueña (Bucher, 1980). La presión antrópica por su parte, incidiría en la diferente cobertura vegetal de los hábitats (Zak \& Cabido, 2002) y, al desaparecer los estratos superiores, en el desarrollo del estrato gramíneo-herbáceo (hasta $0.50 \mathrm{~m}$ ). Ello favoreció a la fauna de tetrápodos -con mayor número de especiesen el hábitat alongado (HA) que tenía esas características (Manzanilla \& Pefaur, 2000).

Esta cobertura mantiene condiciones de temperatura del suelo, humedad ambiental y otras especialmente favorables para el ambiente reproductivo de la herpetofauna (Briguera et al., 2005). En cuanto a los micromamíferos, la importancia de la cobertura herbácea -variable fundamental de su hábitat- habría quedado enmascarada por el análisis conjunto de las comunidades de ambos hábitats (Altrichter et al., 2004; Kufner et al., 2005).

En la distribución de la diversidad de aves según la estructura del hábitat, influyen tanto la cobertura como la altura de la vegetación, siendo esta última fundamental. La estructura vertical de la vegetación fue importante para las comunidades aviares, en ambas estaciones. Durante el periodo húmedo, los estratos superiores reunieron mayor número de especies, muchas de ellas utilizan sólo dichas capas. En el periodo seco, la riqueza de aves se concentraba en los estratos medios, con más cobertura.

Entre las aves, características del hábitat como la altura por encima del suelo y el tipo de sustrato, son usadas de modo diferencial por las especies (Codesido \& Bilenca, 2004). El raleo y la eliminación de estratos de vegetación -en especial los altos- conlleva una pérdida importante en la riqueza de aves de la región. A su vez, alteraciones de la estructura vertical del hábitat boscoso y su transformación en ambientes de borde, colaboran a modificar la composición de especies (Lopez de Casenave et al., 1998). Ello señala la necesidad de mantener el bosque chaqueño y su complejidad estructural.

\section{Literatura citada.}

Adámoli J., Torrella S. \& Ginzburg R. 2004. Gestión Integrada y Desarrollo Sostenible para Reducir la Degradación Social, Económica y Ambiental en el Gran Chaco Americano. Pp. 60-65. En: Informe Dirección de Conservación del Suelo y Lucha contra la Desertificación. Secretaría de Ambiente y Desarrollo Sustentable. Argentina.

Altrichter M., Kufner M., Giraudo L., Gavier G., Tamburini D., Sironi M. \& Arguello L. 2004. Comunidades de micromamíferos de bosque serrano y pastizal de altura en la Sierra Chica, Córdoba, Argentina. Ecología Aplicada. 3 (1, 2): 122-127.

Angelstam P. 1992. Conservation of communities the importance of edges, surroundings and landscape mosaic structure. Páginas 9-70. En: Hansson L. (ed.) Nature conservation by ecological principles - a boreal perspective. Elsevier, London.

Bazán C. 1998. Datos sobre solicitudes de aprovechamiento forestal y desmonte. Dirección de Recursos Renovables y Áreas Naturales, Provincia de Córdoba. En: Kopta F. (ed.) Problemática ambiental con especial referencia a la Provincia de Córdoba. Fundación ACUDE. Córdoba, Argentina. Pp. 6.

Bennet A. 1990. Habitats corridors and the conservation of small mammals in a fragmented forest environment. Landscape Ecology. 4:109-122.

Bibby C., N. Burgess \& Hill D. 1992. Points Counts. Páginas: 85-104. In: Bibby C.J., Burgess N. and Hill D. Bird Census Techniques. Academic Press Limited, San Diego.

Bilenca D. \& Kravetz F. 1995. Patrones de abundancia relativa en ensambles de pequeños roedores de la región pampeana. Ecología Austral. 5: 21-30.

Briguera V., Tamburini D., Kufner M., Gavier G., Giraudo L., Torres R. \& Bechara V. 2006. Herpetofauna en relictos de Bosque Chaqueño de la región de Mar Chiquita, Córdoba. Cuadernos de Herpetología. 20 (1): 25-31.

Bucher E. H. 1980. Ecología de la fauna chaqueña. Una revisión. Ecosur. 7 (14): 111-159.

1990. The Influence of Changes in Regional Land-use Patterns on Zenaida Dove Populations. Pp. 291-303. En: Pinowsky J. and Summers Smith J. (eds.). Granivorous Birds in Agricultural Landscape. Polish Academy of Sciences, Warsaw.

Bucher E. H., Costa Gorriz B. \& Leynaud G. 2001. Bird diversity and forest fragmentation in the semiarid Espinal woodland of Córdoba, Argentina. Bol. Acad. Nac. Ciencias. 66: 117-124.

Cabrera M. 1996. Lista y Distribución de Saurios, Anfisbenas y Tortugas (Amniota, Reptilia) de la Provincia de Córdoba. Páginas 215-238. En: di Tada I. y Bucher E. Biodiversidad de la Provincia de Córdoba. Fauna. Universidad Nacional de Río Cuarto. Córdoba. 2001. Elenco sistemático y claves para la identificación de los reptiles de Córdoba, Argentina (Testudines \& Squamata). Bol. Acad. Nac. Ciencias. 66: 5-24.

Carrascal L. \& Palomino D. 2005. Preferencias de hábitat, densidad y diversidad de las comunidades de aves en Tenerife. Animal Biodiversity and Conservation. 28 (2): 101-119.

CBC-Vertebrados. 2011. Colección Biológica del CERNAR: Vertebrados. UN Córdoba, Argentina. http://www.efn.uncor.edu/investigacion/cernar/coleccion _vertebrados.html.

Cei J. 1980. Amphibians of Argentina. NS Monogr. 2. Italian Journal of Zoology. Pp. 609.

1986. Reptiles del centro, centro-oeste y sur de la Argentina: herpetofauna de las zonas áridas y semiáridas. Museo Regionale di Scienze Naturali. Torino. Pp. 527.

Codesido M. \& Bilenca B. 2004. Variación estacional de un ensamble de aves en un bosque subtropical semiarido del Chaco argentino. Biotrópica. 36 (4): 544-554.

Costa Gorriz B. \& Bucher E. 1995. Diversidad de aves y Fragmentación del bosque Nativo en el NW de la Provincia de Córdoba, Argentina. Tesis de Maestría. UN Córdoba. Argentina. 
Dardanelli, S. \& Nores M. 2001. Extinción y Colonización de Aves en Fragmentos de Bosque de la Provincia de Córdoba, Argentina. Bol. Acad. Nac. Ciencias. 66: 5560.

de Villafañe G., Quintana R., Merler J. \& Bonaventura S. 1994. Selección de hábitat en Akodon azarae y Calomys laucha en semicautividad. Efecto de la densidad de Calomys laucha sobre Akodon azarae. Mastozoología Neotropical. 1 (2): 123-133.

di Tada I., Zavattieri M., Bridarolli M., Salas N. \& Martino A. 1996. Anfibios anuros de la Provincia de Córdoba. Páginas 192-213. En: di Tada I. y Bucher E. (eds). Biodiversidad de la Provincia de Córdoba. Fauna. Universidad Nacional de Río Cuarto. Córdoba.

Fahrig L. 2003. Effects of habitat fragmentation on biodiversity. Annual Reviews of Ecology and Systematics. 34: 487-515.

Gallardo J. 1987. Anfibios Argentinos. Guía para su identificación. Librería Agropecuaria S. A. 1ra. Edición. Pp. 98.

Giannoni S., Borghi C., Dacar M. \& Campos C. 2005. Main food categories in diets of sigmodontine rodents in the Monte (Argentina). Mastozool. Neotropical. 12 (2):181187.

Giraudo L., Kufner M., Torres R., Briguera V. \& Gavier G. 2006. Avifauna del Bosque Chaqueño Oriental de la Provincia de Córdoba, Argentina. Ecología Aplicada. 5 $(1,2)$ : 127-136.

Gonzalez Ittig R. \& Gardenal C. 2004. Recent range expansion and low levels of contemporary gene flow in Calomys musculinus: its relationship with the emergence and spread of Argentine haemorrhagic fever. Heredity. 93: 535-541.

Gordon T. 1997. BioDap: Ecological Diversity and Its Measurement. Resource Conservation Fund National Park, Alma New Brunswick, Canadá.

Hays R., Summers C. \& Willam S. 1981. Estimating Wildlife Habitat Variables. Fish \& Wildlife Service. FWS/OBS-81/47.

Kufner M., Tamburini D., Giraudo L. \& Briguera V. 2005 Conservación de mastofauna en fragmentos de bosque chaqueño en la región de Mar Chiquita (Córdoba, Argentina). Ecología Aplicada. 4 (1,2): 53-58.

Lavilla E., Scrocchi G. \& Avila L. 1992. Clave para la identificación de los anfibios de la Provincia de Córdoba (Argentina). Asociación Herpetológica Argentina, Buenos Aires. Pp. 18.

López de Casanave J., Pelotto J., Caziani S., Mermoz M. \& Protomastro J. 1998. Responses of avian assemblages to a natural edge in Chaco Semiarid Forest in Argentina. The Auk. 115 (2): 425-435.

Luti R., Beltrán de Solis M., Galera F., Müller de Ferreira N., Berzal M., Nores M., Herrera M. \& Barrera J. 1979. Vegetación. Pp. 297-367. En: Vázquez J., Miatello R. y Roque M. (eds.). Geografía Física de la Provincia de Córdoba. Boldt, Buenos Aires.

Magurran A. 1988. Ecological Diversity and its Measurements. Princeton University Press. Pp. 179.

Manzanilla J. \& Péfaur P. 2000. Consideraciones sobre métodos y técnicas de campo para el estudio de anfibios y reptiles. Revista Ecológica de Latino América. 7 (1-2): 17-30.
Matteucci S. \& Colma A. 1982. Metodología para el estudio de la vegetación. Monografía No 22. Secretaría General de la OEA, Washington D. C.

Matteucci S. 1998. La cuantificación de la estructura del paisaje. Pp. 271-291. En: Matteucci S. y Buzai G. (eds.). Sistemas ambientales complejos: herramientas de análisis espacial. Eudeba. Buenos Aires.

Miatello R. 2007. Vertebrados en Extinción de la Provincia de Córdoba. Biológica. 2: 16-22.

Morello J. \& Matteucci S. 1999. Biodiversidad y fragmentación en los Bosques de Argentina. Pp. 463498. En: Matteucci S., Solbrig O., Morello J. y Halffter G. (eds). Biodiversidad y uso de la tierra. Conceptos y ejemplos en Latinoamérica. Eudeba, Buenos Aires.

Narosky T. \& Yzurieta D. 2003. Guía para la identificación de las aves de Argentina y Uruguay. Edición de Oro. Vázquez Manzini, Buenos Aires. Pp. 346.

Peltzer P., Bock G., Tardivo R. \& Lajmanovich R. 2004. Effects of habitat loss and fragmentation on anuran in Espinal Ecoregion, Argentina: GIS approach. Froglog. 63: 3-4.

Peltzer P., Lajmanovich R., Attademo A. \& Cejas W. 2005. Diversidad y conservación de anuros en ecosistemas agrícolas de Argentina: implicancias en el control biológico de plagas. Insugeo. 14: 399-416.

Polop J., Martínez R. \& Torres M. 1985. Distribución y abundancia de poblaciones de pequeños roedores en zona Embalse Río Tercero, Córdoba. Historia Natural. 5 (5): 33-44.

\& Sabattini M. 1993. Rodent Abundance and distribution in habitats of agrocenosis in Argentina. Studies on Neotropical Fauna and Enviromental. 28 (1): 39-46.

\& Provenzal M. 1999. Contribution to the identification of Calomys venustus (Thomas, 1894) (Rodentia, Muridae) by morphological characters. Facena. 15: 163-168.

\& Busch M.C. (eds). 2010. Biología y ecología de pequeños roedores en la región pampeana de Argentina. Enfoques y perspectivas. Universidad Nacional de Córdoba, Córdoba. Pp. 328.

Saunders D., Hobbs R. \& Margules C. 1991. Biological consequences of ecosystem fragmentation: a review. Conservation Biology. 5 (1): 18-25.

Siegel S. 1980. Estadística no paramétrica aplicada a las ciencias de la conducta. Trillas, México D.F. Pp. 346.

Stamatti M., Parmuchi M., Strada M., Bono J., Montenegro C., Manghi E. \& Brouver M. 2004. Mapa Forestal de la Provincia de Córdoba. Dirección de Bosque SAyDS, Buenos Aires. Pp. 16.

Ubeda C. \& Grigera D. 2003. Análisis de la evaluación más reciente del estado de conservación de los anfibios y reptiles de Argentina. Gayana. 67 (1): 97-113.

Zak M. \& Cabido M. 2002. Spatial patterns of the Chaco vegetation of central Argentina: Integration of remote sensing and phytosociology. Applied Vegetation Science. 5: 213-226.

Zuleta G., Kravetz F., Busch M. \& Percich R. 1988. Dinámica poblacional del ratón de pastizal pampeano (Akodon azarae) en ecosistemas agrarios de Argentina. Revista Chilena de Historia Natural. 61: 231-244. 


\section{ANEXOS: tabla y figuras citadas en el texto}

Tabla 2. Comunidades de aves en los hábitats de bosque. Composición y abundancia estacional y gremios tróficos.

\begin{tabular}{|c|c|c|c|}
\hline Nombre específico & $\begin{array}{l}\text { Gremio } \\
\text { trófico }\end{array}$ & $\begin{array}{l}\text { Período } \\
\text { Húmedo }\end{array}$ & $\begin{array}{l}\text { Período } \\
\text { Seco }\end{array}$ \\
\hline Crypturellus tataupa & $\mathrm{O}$ & - & 5 \\
\hline Nothoprocta cinerascens & $\mathrm{O}$ & 4 & - \\
\hline$N$. pentlandii & $\mathrm{H}$ & 1 & - \\
\hline Nothura maculosa & $\mathrm{O}$ & 2 & 7 \\
\hline Coragyps atratus & $\mathrm{Ca}$ & 118 & 55 \\
\hline Geranoaetus melanoleucus & $\mathrm{C}$ & 4 & 4 \\
\hline Polyborus plancus & $\mathrm{C}$ & 20 & 15 \\
\hline Milvago chimango & $\mathrm{C}$ & 6 & 8 \\
\hline Falco peregrinus & $\mathrm{C}$ & 1 & - \\
\hline F. sparverius & $\mathrm{C}$ & 1 & 3 \\
\hline Ortalis canicollis & $\mathrm{O}$ & 1 & 4 \\
\hline Vanellus chilensis & $\mathrm{I}$ & 3 & 6 \\
\hline Columba picazuro & G & 5 & 27 \\
\hline C. maculosa & G & 10 & 35 \\
\hline Zenaida auriculata & G & ------ & ------ \\
\hline Columbina picui & G & 113 & 115 \\
\hline Leptotila verreauxi & G & 2 & 6 \\
\hline Myiopsitta monachus & FG & 15 & 91 \\
\hline Coccyzus melacoryphus & I & 1 & - \\
\hline Guira guira & I & - & 9 \\
\hline Tapera naevia & I & 1 & - \\
\hline Hydropsalis brasiliana & I & 1 & - \\
\hline Sappho sparganura & $\mathrm{N}$ & - & 6 \\
\hline Chlorostilbon aureoventris & $\mathrm{N}$ & 8 & - \\
\hline Nystalus maculatus & $\mathrm{I}$ & 1 & 9 \\
\hline Colaptes melanolaimus & I & 2 & 2 \\
\hline Melanerpes cactorum & I & 3 & - \\
\hline Picoides mixtus & I & - & 1 \\
\hline Picumnus cirratus & I & 1 & 4 \\
\hline $\begin{array}{l}\text { Lepidocolaptes } \\
\text { angustirostris }\end{array}$ & I & 3 & - \\
\hline Upucerthia certhioides & I & - & 12 \\
\hline Furnarius rufus & I & 9 & 6 \\
\hline Coryphistera alaudina & I & - & 6 \\
\hline Cranioleuca pyrrhophia & I & 6 & 7 \\
\hline Asthenes baeri & I & 1 & - \\
\hline Synallaxis frontalis & I & 3 & - \\
\hline S. albescens & I & 6 & - \\
\hline Pseudoseisura lophotes & I & 10 & - \\
\hline Leptasthenura platenses & I & - & 1 \\
\hline Thamnophilus caerulescens & I & 3 & 2 \\
\hline Taraba major & I & 2 & 1 \\
\hline Rhinocrypta lanceolata & I & 5 & - \\
\hline Melanopareia maximiliani & I & 1 & - \\
\hline Phytotoma rutila & $\mathrm{H}$ & 1 & 1 \\
\hline Xolmis irupero & $\mathrm{I}$ & 2 & 1 \\
\hline Stigmatura budytoides & I & - & 4 \\
\hline Pitangus sulphuratus & $\mathrm{O}$ & 3 & 1 \\
\hline $\begin{array}{l}\text { Empidonomus } \\
\text { aurantioatrocristatus }\end{array}$ & I & 1 & - \\
\hline Suiriri suiriri & I & 1 & - \\
\hline Myiophobus fasciatus & I & 2 & - \\
\hline $\begin{array}{l}\text { Todirostrum } \\
\text { margaritaceiventer }\end{array}$ & I & 3 & 3 \\
\hline Euscarthmus meloryphus & I & 4 & - \\
\hline
\end{tabular}


Serpophaga subcristata Elaenia albiceps

E. parvirostris

Phaeoprogne tapera

Stelgidopteryx fucata

Troglodytes aedon

Anthus sp.

Mimus triurus

Turdus amaurochalinus

T. chiguanco

Polioptila dumicola

Cyclarhis gujanensis

Thraupis bonariensis

Saltator aurantiirostris

Catamenia analis

Sicallis luteola

S. flaveola

Coryphospingus cucullatus

Aimophila strigiceps

Zonotrichia capensis

Saltatricula multicolour

Poospiza nigrorufa

P. torquita

P. melanoleuca

Embernagra platenses

Carduelis magellanica

Molothrus rufoaxillaris

M. bonariensis

M. badius

Total de Individuos

Número de Especies

Referencias: H Herbívoro; G Granívoro; FG Frugi-granívoro;

N Nectarívoro; O Omnívoro; I Insectívoro; C Carnívoro y Ca

Carroñero.

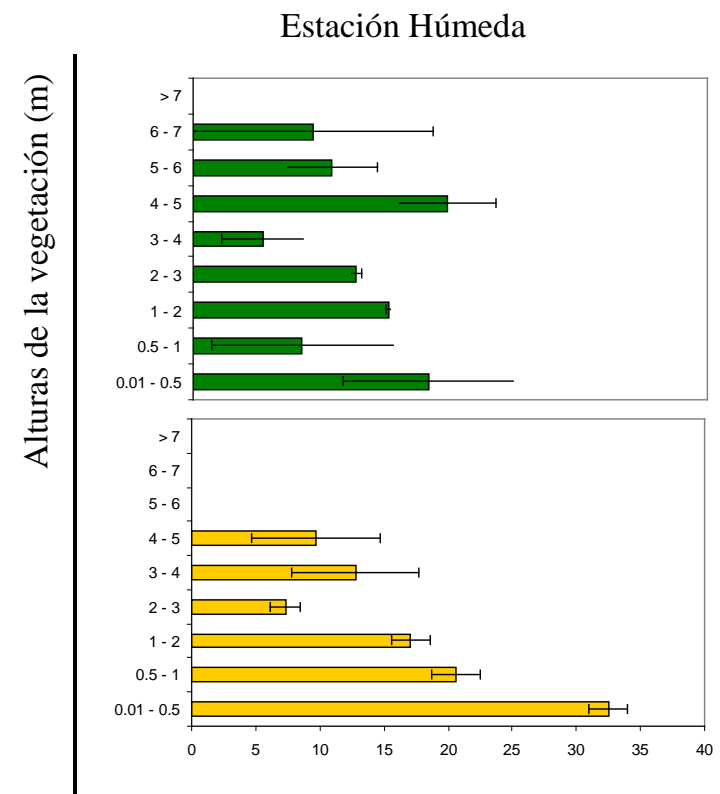

Estación Seca

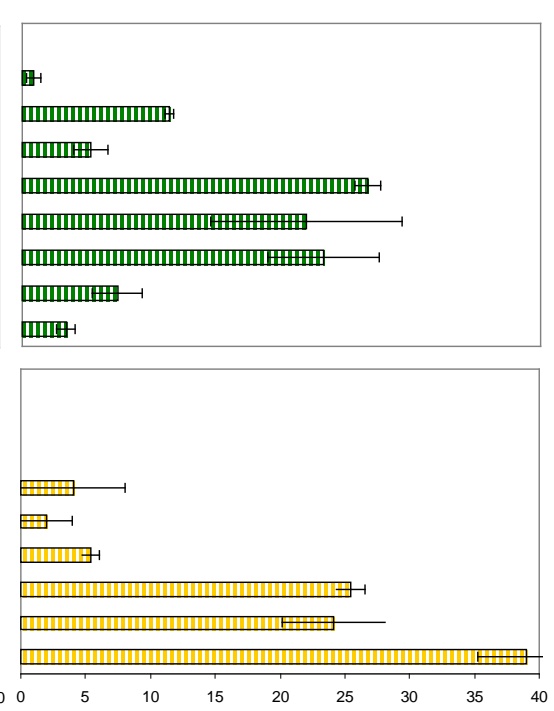

Cobertura Vegetal (\%)

Figura 1. Estructura de la vegetación en Las Peñas, Dpto. Totoral. Cobertura vegetal promedio de los intervalos de altura y desviación estándar. Referencias: Hábitat HC en verde y Hábitat HA en amarillo. 


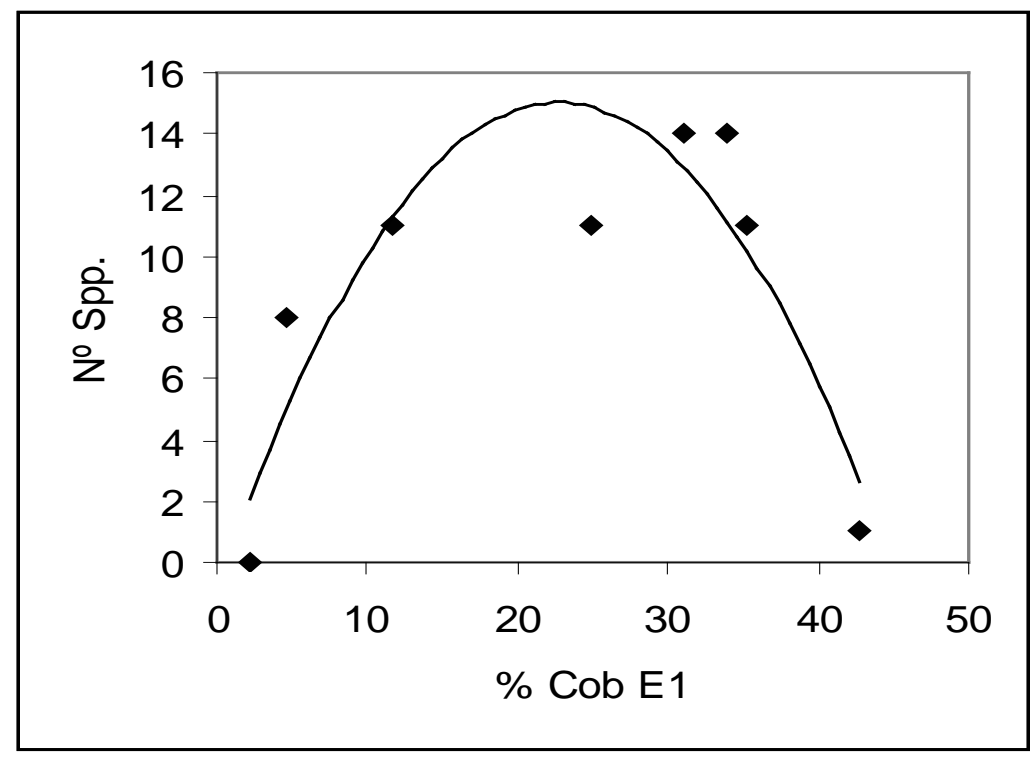

Figura 2. Distribución de las especies de tetrápodos del suelo en relación a la cobertura herbácea.

\footnotetext{
${ }^{1}$ Universidad Nacional de Córdoba (Argentina), Av. Vélez Sarsfield 1611, dirección electrónica de correspondencia: bkufner@com.uncor.edu
} 